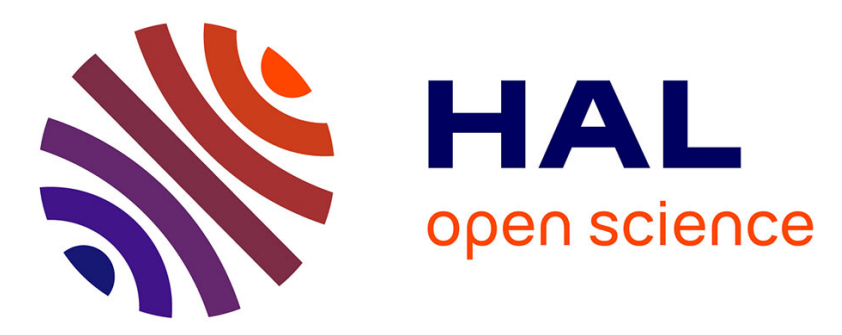

\title{
Direct temporal reconstruction of a picosecond pulse by cross-correlation in a semiconductor device
}

Charles-Henri Hage, Franck Billard, Bertrand Kibler, Christophe Finot, Guy Millot

\section{- To cite this version:}

Charles-Henri Hage, Franck Billard, Bertrand Kibler, Christophe Finot, Guy Millot. Direct temporal reconstruction of a picosecond pulse by cross-correlation in a semiconductor device. Electronics Letters, 2012, 48 (13), pp.778-780. 10.1049/el.2012.1608 . hal-00703307

\section{HAL Id: hal-00703307 https://hal.science/hal-00703307}

Submitted on 1 Jun 2012

HAL is a multi-disciplinary open access archive for the deposit and dissemination of scientific research documents, whether they are published or not. The documents may come from teaching and research institutions in France or abroad, or from public or private research centers.
L'archive ouverte pluridisciplinaire HAL, est destinée au dépôt et à la diffusion de documents scientifiques de niveau recherche, publiés ou non, émanant des établissements d'enseignement et de recherche français ou étrangers, des laboratoires publics ou privés. 


\section{Direct temporal reconstruction of a picosecond pulse by cross-correlation in a semiconductor device}

\section{C.-H. Hage, F. Billard, B. Kibler, C. Finot and G. Millot}

Cross-correlation measurements using the two-photon absorption process in a semiconductor is experimentally demonstrated for two pulses of different wavelengths (shifted by $\sim 200 \mathrm{~nm}$ ) and durations (20 times ratio). These measurements were found to be highly repeatable and fully suitable for the determination of the temporal intensity profile of picosecond (ps) pulses.

Introduction: Ultrashort optical pulses are nowadays a basic requirement for numerous applications such as optical communications, nonlinear spectroscopy or terahertz field [1]. As measurement and control of these pulses cannot be achieved by electronic means, alloptical methods have been developed. For example, temporal intensity profiles can be reconstructed from cross correlation records based on an interferometric device which measures the correlation between an unknown pulse and a reference ultrashort pulse through their product in a nonlinear crystal. Although these methods are accurate, a delicate trade-off has to be found between efficiency and spectral bandwidth [2]. This drawback can be partly circumvented by using the two-photon absorption (TPA) process in a semiconductor which permits wideband and ultrashort pulses measurements [2-4], the process being only limited by the peak power of the pulses [4].

Optical cross-correlation between pulses widely different in terms of central wavelength and pulse duration $(\Delta \lambda>100 \mathrm{~nm}$ and $\Delta \mathrm{T}$ ratio $>10)$ has been demonstrated in nonlinear crystals [5] but to the best of our knowledge, it has never been reported in a semiconductor device. Here we present what we believe to be the first cross-correlation between two completely different pulses in a semiconductor device.

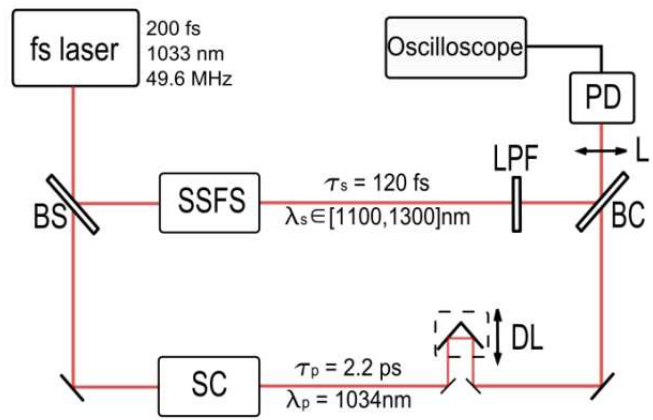

Fig. 1 Experimental setup. $B S=$ Beam-Splitter, SSFS $=$ Soliton SelfFrequency Shift, $S C=$ Spectral Compression, $L P F=$ Long Pass Filter, $D L=$ optical Delay Line, $B C=$ Beam Combiner,$P D=$ PhotoDiode

Experimental setup and results: The experimental setup is depicted in Fig. 1. A femtosecond (fs) laser (Amplitude Systèmes tPulse model, central wavelength of $1033 \mathrm{~nm}$, pulse duration of $180 \mathrm{fs}$, repetition rate of $49.6 \mathrm{MHz}$, average power of $1 \mathrm{~W}$ ) is divided in two by a beamsplitter (BS). In the first part of the setup, wavelength tunable pulses are created using the Raman soliton self-frequency shift (SSFS) process [6] occurring in a highly non-linear photonic crystal fibre with anomalous dispersion. These pulses are Fourier transform limited and have a nearly-constant full-width at half-maximum (FWHM) duration $\tau_{\mathrm{s}}=120 \mathrm{fs}$ (details of the experimental implementation and results of the SSFS block are provided in [7]). A long-pass filter (LPF) is used to remove the pump residue. A second block of the setup is based on the spectral compression (SC) process [8] that enables the generation of ps pulses with a spectral width of $\sim 1 \mathrm{~nm}$ (such a spectral narrowing is experienced upon the nonlinear propagation in optical fibre of chirped pulses, details of the block are not provided here). These two pulses are spatially recombined by a dichroic beam-combiner (BC) and their temporal overlap is controlled by means of an optical delay line (DL).
The pulses are collinearly polarized in order to avoid the influence of the anisotropy of the photodiode semiconductor nonlinear absorption [9] and are tightly focused on a GaAsP photodiode (PD, Hammamatsu G1116 model). The resulting photocurrent is monitored by an oscilloscope with high input impedance $(1 \mathrm{M} \Omega)$. In order to get a reasonable optical signal to noise ratio, we used pulse energies of about $30 \mathrm{pJ}$ although lower energy levels $(10 \mathrm{pJ})$ are sufficient.

We first study cross-correlation measurements involving ultrashort solitons shifted (by SSFS) to $1150 \mathrm{~nm}$. The resulting signal is shown in Fig. $2 a$. As the fs pulse is more than one order of magnitude shorter than the ps one, it can be in first approximation considered as a Dirac function for the convolution operation. In other words, the crosscorrelation record should reproduce directly the temporal intensity profile of the ps pulse [1]. We have checked this assumption by computing autocorrelation signal when considering the intensity profile of the ps pulse being equal to the cross-correlation signal (Fig $2 b$, circles) and we have experimentally compared the result with an independently measured autocorrelation signal (solid line, measurements made with a Femtochrome FR-103HS device based on second harmonic generation in a nonlinear crystal). The two autocorrelation signals have been found in excellent agreement, which validates that given the very different temporal durations under investigation, there is no need of carrying out complex deconvolution processes to take into account the temporal extend of the shortest pulse. We can therefore estimate directly the FWHM of the pulse, i.e. $2.2 \mathrm{ps}$, without assuming any shape factor as usually required for measurements based on autocorrelation signals. The two independently measured signals have also confirmed that no temporal substructure was present in the picosecond pulse.

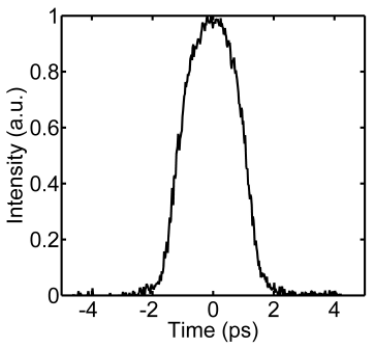

$a$

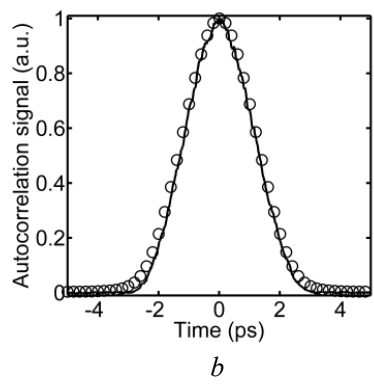

Fig.2 Cross-correlation results.

a Cross-correlation signal (result is averaged over 10 independent measurements)

$b$ Auto-correlation computed from the temporal intensity profile inferred from the cross-correlation record (circles) and compared with an independently measured autocorrelation signal of the ps pulse.

In Fig. $3 a$ we further check that the fs pulse can be considered as a Dirac function for the cross-correlation operation. We numerically simulated cross-correlation signals between the ps pulse and pulses of different durations (from $1 \mathrm{ps}$ down to $10 \mathrm{fs}$ ) and in order to quantify our error, we compute two relevant parameters. The first one is the error made in estimating the FWHM duration directly from the crosscorrelation signal. We can see that a fs pulse duration of less than half the ps one leads to an error below $5 \%$, which means that the duration parameter is not critically affected by the ratio between the pulses durations (as long as the intensity profiles under test are not too complex and do not present ultrashort substructures). The second parameter we use is a misfit parameter [10] aimed at quantifying the error between the temporal intensity profile $I(t)$ of the long pulse and the cross-correlation signal $\mathrm{X}(\mathrm{t})$. It can therefore be defined as :

$$
M^{2}=\int[I(t)-X(t)]^{2} d t / \int I(t)^{2} d t
$$

We can make out from Fig. $3 a$ that this parameter is much more sensitive than the FWHM duration: pulse durations less than $\tau_{\mathrm{p}} / 10$ are required in order to reach a $5 \%$ misfit. As our fs pulse is about $\tau_{\mathrm{p}} / 20$ it is far short enough to fit our needs. 


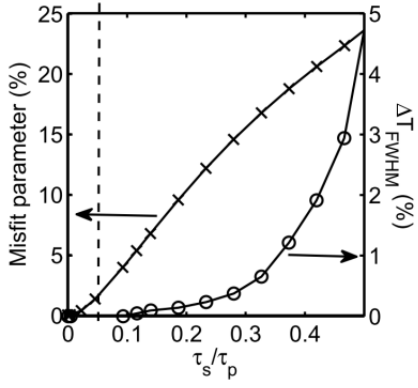

$a$

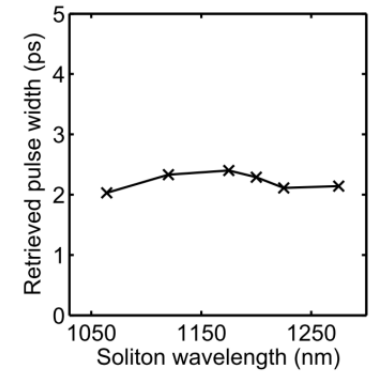

$b$
Fig3 Direct and broadband temporal reconstruction. a Numerical analysis: influence of the ratio of pulse durations involved in the cross-correlation. Error on the FWHM temporal duration of the ps pulse (circles, right axis) and misfit parameter (crosses, left axis). The vertical dashed line indicates the ratio used in our experiments.

$b$ Experiments: temporal pulse duration (FWHM) obtained from crosscorrelation measurements for different fs pulses wavelengths.

Finally to assess the "broadband" aspect of our setup we made crosscorrelation measurements for different fs pulse wavelengths (the femtosecond pulse wavelength is easily tuned by varying the optical peak power at the input of the SSFS block $[6,7])$. The results are shown in Fig. $3 b$ and exhibit as expected a nearly constant pulse duration. Note that our method is here limited by the maximum redshift of the SSFS block. By using the same photodiode, the TPA process is expected to work with ultrashort shifted solitons up to $1700 \mathrm{~nm}$.

Conclusion: We have experimentally demonstrated direct and broadband temporal reconstruction of picosecond pulses based on crosscorrelation measurements in a semiconductor device. By considering pulses with quite distinct temporal durations we overcome the need of deconvolution algorithms and the required details of the shortest pulse. In our configuration where the ps pulse under investigation does not present rapid fluctuations, the cross-correlation can indeed be considered as reproducing directly the temporal intensity profile of the longer pulse. The method has been found robust compared to the wavelength spacing between the two pulses and the measurements have been highly repeatable. It can be considered as a simple, clean, reliable and highly integrable multipurpose tool to synchronize beams, measure jitters or doing accurate picosecond pulse measurements.

Acknowledgments: We acknowledge the support of the Agence Nationale de la Recherche (ANR SOFICARS) through the grant ANR07-RIB-013-03. C.-H. Hage acknowledges the support of the Conseil Régional de Bourgogne through his grant "Jeune Chercheur Entrepreneur" scheme. B. Kibler also thanks the support of the Conseil Régional de Bourgogne through the Photcom PARI grant.

C.-H. Hage, F. Billard, B. Kibler, C. Finot and G. Millot (Laboratoire Interdisciplinaire Carnot de Bourgogne, UMR 6303 CNRS-Université de Bourgogne, Dijon 21078, France)

E-mail: charles-henri.hage@u-bourgogne.fr

\section{References}

1. Kaizer, W.: 'Ultrashort laser pulses, generation and applications, Second Edition' (Springer Verlag, 1993)

2. Ranka, J.K., Gaeta, A.L., Baltuska, A., Pshenichnikov, M.S., and Wiersma, D.A.: 'Autocorrelation measurement of 6-fs pulses based on the two-photon-induced photocurrent in a GaAsP photodiode', Opt. Lett, 1997, 22, (17), pp. 1344-1346

3. Laughton, F.R., Marsh, J.H., Barrow, D.A., and Portnoi, E.L.: 'The two-photon absorption semiconductor waveguide autocorrelator', IEEE Jal. of Qtum. Elec., 1994, 30, (3), pp. 838-845

4. Krug, T., Lynch, M., Bradley, A.L., Donegan, J.F., Barry, L.P., Folliot, H., Roberts, J.S., and Hill, G.: 'High-Sensitivity Two-Photon
Absorption Microcavity Autocorrelator', IEEE Ph. Technol. Lett., 2004, 16, (6), pp. 1543-1545

5. Deng, Y., Yang, H., Tang, M., Fu, X., and Wen, S.: 'Experimental research on measuring the fine structure of long pulse in time domain by synchronized ultrashort pulse', Opt. Com., 2011, 284, pp. 847-851 6. Mitschke, F.M., and Mollenauer, L.F.: 'Discovery of the soliton selffrequency shift, Opt. Lett., 1986, 11, (10), pp. 659-661

7. Hage, C.H., Kibler, B., Andresen, E.R., Michel, S., Rigneault, H., Courjaud, A., Mottay, E., Dudley, J.M., Millot, G., and Finot, C.: 'Optimization and characterization of a femtosecond tunable light source based on the soliton self-frequency shift in photonic crystal fiber' Proc. SPIE, 2011, 8071, (8071 0I)

8. Planas, S.A., Pires Mansur, N.L., Brito Cruz, C.H., and Fragnito, H.L.: 'Spectral narrowing in the propagation of chirped pulses in singlemode fibers', Opt. Lett., 1993, 18, (9), pp. 699-701

9. Santran, S., Martínez-Rosas, M.E., Canioni, L., and Sarger, L.: 'Characterization of Optical Nonlinearity in Semiconductor Photodiodes Using Cross-Polarized Autocorrelation', IEEE Jal. of Qtum. Elec., 2004, 40, (12), pp. 1687-1694

10. Finot, C., Parmigiani, F., Petropoulos, P., and Richardson, D.J.: 'Parabolic pulse evolution in normally dispersive fiber amplifiers preceeding the similariton formation regime', Opt. Expr., 2006, 14, (8), pp. 3161-3170 